\title{
SWOG S0800 (NCI CDR0000636131): addition of bevacizumab \\ to neoadjuvant nab-paclitaxel with dose-dense doxorubicin and cyclophosphamide improves pathologic complete response (pCR) rates in inflammatory or locally advanced breast cancer
}

\author{
Z. A. Nahleh ${ }^{1}$ (D) W. E. Barlow ${ }^{2}$ - D. F. Hayes ${ }^{3}$ - A. F. Schott ${ }^{3}$ J. R. Gralow ${ }^{4}$

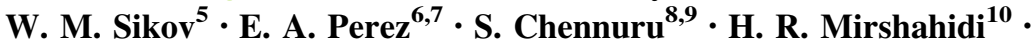 \\ S. W. Corso ${ }^{11}$ - D. L. Lew ${ }^{2}$ - L. Pusztai ${ }^{12}$ - R. B. Livingston ${ }^{13}$ - G. N. Hortobagyi ${ }^{14}$ \\ Received: 23 June 2016/Accepted: 25 June 2016/Published online: 8 July 2016 \\ (c) The Author(s) 2016. This article is published with open access at Springerlink.com
}

\begin{abstract}
SWOG S0800, a randomized open-label Phase II clinical trial, compared the combination of weekly nab-paclitaxel and bevacizumab followed by dose-dense doxorubicin and cyclophosphamide (AC) with nab-paclitaxel followed or preceded by $\mathrm{AC}$ as neoadjuvant treatment for HER2-negative locally advanced breast cancer (LABC) or inflammatory breast cancer (IBC). Patients were randomly allocated (2:1:1) to three neoadjuvant chemotherapy arms: (1) nab-paclitaxel with concurrent bevacizumab followed by AC; (2) nab-paclitaxel followed by AC; or (3) AC followed by nab-paclitaxel. The primary endpoint was pathologic complete response (pCR) with stratification by disease type (non-IBC LABC vs. IBC) and hormone receptor status (positive vs. negative). Overall survival (OS), event-free survival (EFS), and toxicity were secondary endpoints. Analyses were intent-to-treat comparing bevacizumab to the combined control arms. A total of 215 patients were accrued including $11 \%$ with IBC and $32 \%$ with triple-negative breast cancer (TNBC). The addition of bevacizumab
\end{abstract}

$\triangle$ Z. A. Nahleh

zeina.nahleh@ttuhsc.edu

1 Division of Hematology-Oncology, Department of Internal Medicine, Texas Tech University Health Sciences Center, Paul L. Foster School of Medicine, El Paso, TX, USA

2 SWOG Statistical Center, Seattle, WA, USA

3 University of Michigan, Ann Arbor, MI, USA

4 Seattle Cancer Care Alliance, University of Washington, Seattle, WA, USA

5 Women and Infants Hospital of Rhode Island and Warren Alpert Medical School of Brown University, Providence, RI, USA

6 Genentech, Inc., San Francisco, CA, USA

7 Mayo Clinic, Jacksonville, FL, USA significantly increased the pCR rate overall (36 vs. $21 \%$; $p=0.019)$ and in TNBC (59 vs. $29 \% ; p=0.014$ ), but not in hormone receptor-positive disease (24 vs. $18 \%$; $p=0.41)$. Sequence of administration of nab-paclitaxel and AC did not affect the pCR rate. While no significant differences in OS or EFS were seen, a trend favored the addition of bevacizumab for EFS $(p=0.06)$ in TNBC. Overall, Grade 3-4 adverse events did not differ substantially by treatment arm. The addition of bevacizumab to nab-paclitaxel prior to dose-dense AC neoadjuvant chemotherapy significantly improved the $\mathrm{pCR}$ rate compared to chemotherapy alone in patients with triple-negative LABC/IBC and was accompanied by a trend for improved EFS. This suggests reconsideration of the role of bevacizumab in high-risk triplenegative locally advanced breast cancer.

Keywords Breast cancer - Locally advanced . Inflammatory $\cdot$ Bevacizumab $\cdot$ Neoadjuvant

8 Hematology Oncology Consultants, Inc., Westerville, $\mathrm{OH}$, USA

9 Columbus NCI Community Oncology Research Program, Columbus, OH, USA

10 Loma Linda University Cancer Center, Loma Linda, CA, USA

11 Gibbs Cancer Center and Research Institute/Southeast Clinical Oncology Research (SCOR) Consortium NCORP/ Upstate Carolina CCOP (previous), Spartanburg, SC, USA

12 Yale University, New Haven, CT, USA

13 Arizona Cancer Center, Tucson, AZ, USA

14 University of Texas MD Anderson Cancer Center, Houston, TX, USA 


\section{Introduction}

Locally advanced breast cancer (LABC) was historically defined as cancers that were inoperable, T4 and/or advanced regional nodal disease at presentation, and had poor survival outcomes with locoregional therapy alone. However, the definition has expanded to include potentially operable tumors greater than $5 \mathrm{~cm}$ [1-3]. Inflammatory breast cancer (IBC) characterized by diffuse erythema or edema of the affected breast, with or without histologically confirmed involvement of the dermal lymphatics, is a highly aggressive form of $\mathrm{LABC}$ that has poor prognosis, with 10-year disease-free survival rates reported at 20-25\% [4]. Few randomized studies have targeted LABC and/or IBC and most large adjuvant and neoadjuvant trials exclude these patients. Anthracycline- and taxane-based neoadjuvant chemotherapy represents the standard of care for LABC. Pathologic complete response (pCR), commonly defined as the absence of residual invasive cancer in both the breast and axillary lymph nodes, has emerged as a surrogate endpoint for disease-free and overall survival, as the achievement of a pCR is associated with a favorable long-term prognosis in all breast cancer subtypes, while extensive residual disease predicts for poor outcomes, especially in triple-negative [estrogen receptor (ER), progesterone receptor $(\mathrm{PgR})$ and human epidermal growth factor receptor 2 (HER2) negative] and HER2-positive breast cancers [5-7].

Angiogenesis is believed to play a significant role in LABC/IBC [8-12]. Bevacizumab is a recombinant, humanized, monoclonal antibody that binds and neutralizes the vascular endothelial growth factor A, thus acting as an antiangiogenic agent. Bevacizumab has activity in multiple advanced neoplasms, including breast cancer [12-17]. However, after initial enthusiasm over the combination of bevacizumab with chemotherapy in the metastatic setting, subsequent analyses suggested that bevacizumab produces more toxicity than benefit, and initial accelerated approval for the drug in this setting was subsequently withdrawn by the Food and Drug Administration (FDA) [18]. In addition, two prospective randomized trials failed to document benefit for the addition of bevacizumab to adjuvant chemotherapy in any subset of breast cancer patients $[19,20]$.

However, in the initial metastatic trials, bevacizumab showed activity in some patients when administered with chemotherapy [21]. Despite suggestions that patients with ER/PgR-positive and those with triple-negative cancers may benefit from the addition of bevacizumab [25-28]; retrospective analyses have failed to identify predictive biomarkers that might permit more efficient use of this agent [22]. Therefore, in the current trial (SWOG0800-
ClinicalTrials.gov NCT00856492), we prospectively examined whether neoadjuvant bevacizumab might be more active within selected intrinsic subtypes when administered with neoadjuvant chemotherapy. Furthermore, the vascular pruning hypothesis proposed by Jain suggests that antiangiogenesis might improve flow and oxygenation and enhance the delivery and proapoptotic effect of certain chemotherapy agents, in particular the taxanes $[17,23]$. Thus, we considered whether the addition of bevacizumab to neoadjuvant weekly nab-paclitaxel followed by dose-dense doxorubicin and cyclophosphamide ("AC") would increase the pCR rates in patients with HER2-negative LABC/IBC. Nab-paclitaxel was chosen as the taxane backbone based on several advantages compared to paclitaxel at the time of trial initiation, including increased intratumoral drug levels [24], albumin-mediated receptor transport of the drug via secreted protein acidic and rich in cysteine (SPARC)/osteonectin [25] overexpressed in around $55 \%$ of primary breast tumors [26], specific receptor-mediated transport mechanisms due to overexpression of caveolin-1 and -2 in IBC [27], and antiangiogenic activity as well as synergistic activity with antiangiogenic agents [28, 29]. More recently, Nab-paclitaxel was shown to be more effective than conventional paclitaxel as part of a neoadjuvant regimen for patients with high-risk early breast cancer in a large German study, the GeparSepto [30]. This study found that $38 \%$ of patients who received $n a b$-paclitaxel during the randomized phase III trial achieved a pCR, compared with $29 \%$ of participants who were given conventional paclitaxel, $p<0.001$.

\section{Patients and methods}

\section{Patient population and selection criteria}

Eligible patients were women with biopsy-confirmed, previously untreated, clinical stage IIB to IIIC HER-2negative breast carcinoma and known hormone receptor status. HER-2 status was determined locally according to the 2007 American Society of Clinical Oncology/College of American Pathology guidelines [31]. The clinical diagnosis of IBC (T4d) was based on AJCC cancer staging criteria. Patients had to have a Zubrod Performance Status of $0-2$ and adequate hematologic, renal, and hepatic function. Patients over the age of 60 or with a history of hypertension were required to have a normal echocardiogram or multigated acquisition scan (MUGA). Patients were not permitted to have pre-existing peripheral neuropathy grade $>2$, be pregnant or nursing, or have a history of a cerebrovascular accident, transient ischemic attack, or cardiac event within 12 months prior to registration. 


\section{Treatment plan}

Figure 1 illustrates the treatment schema. Patients were randomly assigned to Arm 1, 2, or 3 in a 2:1:1 ratio according to a dynamic allocation scheme based on two stratification factors (1) IBC vs. not and (2) hormone receptor-positive $(\mathrm{ER} / \mathrm{PgR}+)$ vs. triple-negative breast cancer (TNBC).

Patients randomized to treatment on Arm 1 (bevacizumab) received intravenous (IV) administration of nabpaclitaxel $100 \mathrm{mg} / \mathrm{m}^{2} \mathrm{IV}$ weekly for 12 weeks $(\mathrm{nP} \times 12)$ with IV bevacizumab $10 \mathrm{mg} / \mathrm{kg}$ every 2 weeks (six doses), followed by IV doxorubicin $60 \mathrm{mg} / \mathrm{m}^{2}$ and cyclophosphamide $600 \mathrm{mg} / \mathrm{m}^{2}$ with pegfilgrastim $6 \mathrm{mg}$ subcutaneously every 2 weeks for six cycles $(\mathrm{ddAC} \times 6)$. Patients randomized to Arm 2 received $\mathrm{nP} \times 12$ followed by ddAC $\times 6$, and those randomized to Arm 3 received ddAC $\times 6$ first followed by $\mathrm{nP} \times 12$, both without bevacizumab. The use of six cycles of AC was based on a similar therapy duration used in a parallel adjuvant SWOG study S0221 [32]. At the time when the study was initiated, the use of six cycles of doxorubicin and cyclophosphamide was common in breast cancer clinical trials [33, 34]. The ideal duration of adjuvant doxorubicin chemotherapy for patients with breast cancer, especially those with $\mathrm{LABC}$, is not known. The results of the Cancer and Leukemia Group B trial 40101 conducted using a phase III factorial design, to define whether six cycles of a chemotherapy regimen are superior to four cycles, were not yet known [Shulman, 2014\#5657]. The dose of nab-paclitaxel was chosen based on studies at the time showing that weekly administration of $100 \mathrm{mg} / \mathrm{m}^{2}$ nab-paclitaxel as single agent showed the same antitumor activity as $125 \mathrm{mg} / \mathrm{m}^{2}$, with the benefit of a more favorable toxicity profile in patients with advanced disease [35]. NCI Common Terminology Criteria for Adverse Events (CTCAE) Version 4.0 was utilized for adverse event reporting and toxicity monitoring. The bevacizumab dose was never reduced, but treatment was held for uncontrolled hypertension or any grade 3 toxicity attributed to this agent and permanently discontinued for grade 3 hypertension not controlled medically. If the study treatment was interrupted for more than 3 consecutive weeks, the patient was taken off the study.

\section{Surgical management}

Surgery was performed within 3-6 weeks after completion of neoadjuvant chemotherapy. Patients with LABC who had an excellent clinical response could undergo breastconserving surgery, but mastectomy was required for patients with IBC regardless of their response to treatment. Postneoadjuvant axillary staging was required for all patients. In clinical N0 patients, a sentinel node (SN) biopsy procedure was allowed; in patients who were still clinically node-positive and those with a positive SN biopsy, a full axillary lymph node dissection was required. Patients who progressed on study treatment were removed from protocol treatment. Postlumpectomy and postmastectomy standard breast radiation therapy (RT) was required irrespective of pathological response [36].

\section{Pathologic evaluation}

Pathologic response was determined by local pathologists who were instructed on the study definition of pCR. Surgical pathology reports were reviewed centrally for accuracy of coding by the study chair (Z.N.) without the knowledge of treatment assignment. pCR was defined as
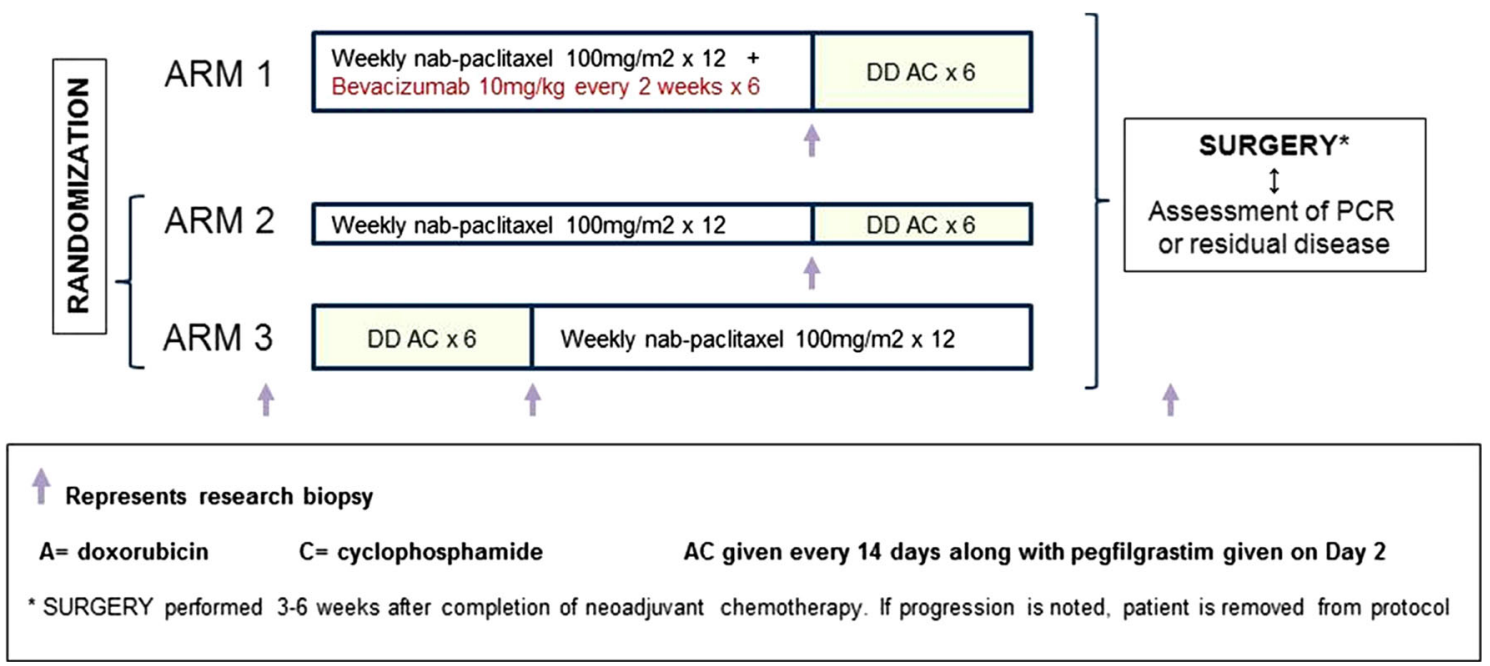

Fig. 1 Schema of randomized Phase II SWOG S0800 trial 
the absence of residual invasive disease with or without ductal carcinoma in situ (ypT0/isN0) in breast and axilla. Specific procedures were provided for evaluation of surgical specimens following neoadjuvant therapy (Appendix).

\section{Statistical analysis}

SWOG S0800 was a randomized Phase II trial comparing the experimental bevacizumab arm (Arm 1) with the control (no bevacizumab) arms (Arms 2 and 3). Accrual was expected to take 2 years with a planned follow-up of two additional years. Based on SWOG study S0012 with similar patients and similar chemotherapy backbone [37], we estimated that the control pCR rate would be $25 \%$, and that a sample size of 200 would allow detection of an increase of $15 \%$ in pCR rate to $40 \%$. Power was $80 \%$ (1sided $\alpha=0.10$ ) for the primary comparison of bevacizumab versus no bevacizumab (collapsing over the two sequences). Dynamic balancing was used to adjust the randomization probabilities so that patient allocation was balanced within each stratum. Near the end of the trial an incorrect adjustment by SWOG programming staff disturbed the dynamic balancing allocation, causing more patients to be assigned to Arm 2 and fewer to Arm 3 than intended. However, this did not affect the primary comparison of $\mathrm{pCR}$ rates between bevacizumab versus no bevacizumab. Analysis was intent-to-treat of eligible patients which is the SWOG standard approach [38].

The secondary randomization of sequence between Arms 2 and 3 was conducted to allow study of the impact of potential predictive biomarkers such as SPARC proteins. There was no expectation of a difference in pCR due to the sequencing, and the primary statistical plan was to compare the intervention arm to the combined control arms. Predictive longitudinal biomarker studies are being conducted comparing all three arms and will be reported separately [39].

Overall survival (OS) was a secondary endpoint defined as the time from registration to death due to any cause. We also analyzed event-free survival (EFS) starting at the time of registration. Events included progression prior to surgery, recurrence postsurgery, or death from any cause. Patients without an event were censored at the last known follow-up time. OS and EFS were analyzed using stratified log-rank tests and Cox regression.

The third objective was to explore for an interaction between bevacizumab and the stratification factors on pCR. A Cochran-Mantel-Haenszel test was performed for the primary analysis comparing pCR rates between the bevacizumab and no bevacizumab groups adjusting for the stratifying variables. We then used logistic regression to explore for an interaction of treatment with type of disease and ER/PgR status. The SWOG Data and Safety Monitoring Committee reviewed the study every 6 months for safety, but no interim analyses were planned or conducted.

\section{Results}

\section{Patient characteristics}

Between May 2010 and September 2012, 215 patients from SWOG member institutions were enrolled and randomized. Two patients were deemed ineligible due to clinical Stage IIA disease and two more withdrew consent leaving 211 patients for analysis. The final allocation of analyzable patients was 98, 62, and 51 in Arms 1, 2, and 3, respectively (Fig. 2).

Baseline characteristics of the patients and their tumors are provided in Table 1 . The vast majority of the participants had non-inflammatory LABC (89\%), whereas only $11 \%$ had IBC. Sixty-eight percent of tumors were ER/ $\mathrm{PgR}+$.

\section{Primary outcome}

Table 2 shows pCR rates by randomized group and predefined patient subsets. Seventeen $(8 \%)$ patients had either no definitive surgery $(n=15)$ or an incomplete pathology report $(n=2)$, and were coded as no pCR in the intentionto-treat analyses. At the time of surgery, 135 (64\%) of the 211 patients had residual invasive disease (no pCR) and 59 (28\%) patients achieved a pCR. Overall, the pCR rate was significantly higher in patients who received bevacizumab 36 vs. $21 \%$ for the non-bevacizumab arms; stratified $p=0.019$ ). In $\mathrm{ER} / \mathrm{PgR}+$ disease (defined as $\geq 1 \%$ expression by immunohistochemistry (IHC) stain), there was no statistically significant difference (bevacizumab $24 \%$ vs. non-bevacizumab $18 \% ; p=0.41)$, whereas the pCR rate was statistically superior with bevacizumab in TNBC (bevacizumab $59 \%$ vs. non-bevacizumab $29 \%$; $p=0.014)$. In non-IBC, the overall pCR rate was $29 \%$ with a higher rate in the patients treated with bevacizumab (36 vs. $22 \% ; p=0.037$ ). A higher $\mathrm{pCR}$ rate for bevacizumab-treated patients with IBC (30 vs. $14 \%$ ) was not statistically significant in this small patient subset $(p=0.61)$. In a multivariate logistic regression analysis, the increase in the pCR rate with bevacizumab was statistically significant ( $p=0.023$ ) adjusting for the type of disease (IBC vs. not; ER/PgR+ vs. TNBC) and neither significantly interacted with treatment $(p=0.62$ and $p=0.19$, respectively). In the non-bevacizumab arms, pCR rates did not differ by treatment sequence (Arm 2 $23 \%$, Arm $320 \%, p=0.82$ ), justifying merging these two control groups. Of the 195 patients who had surgery 
Fig. 2 Consort diagram for S0800

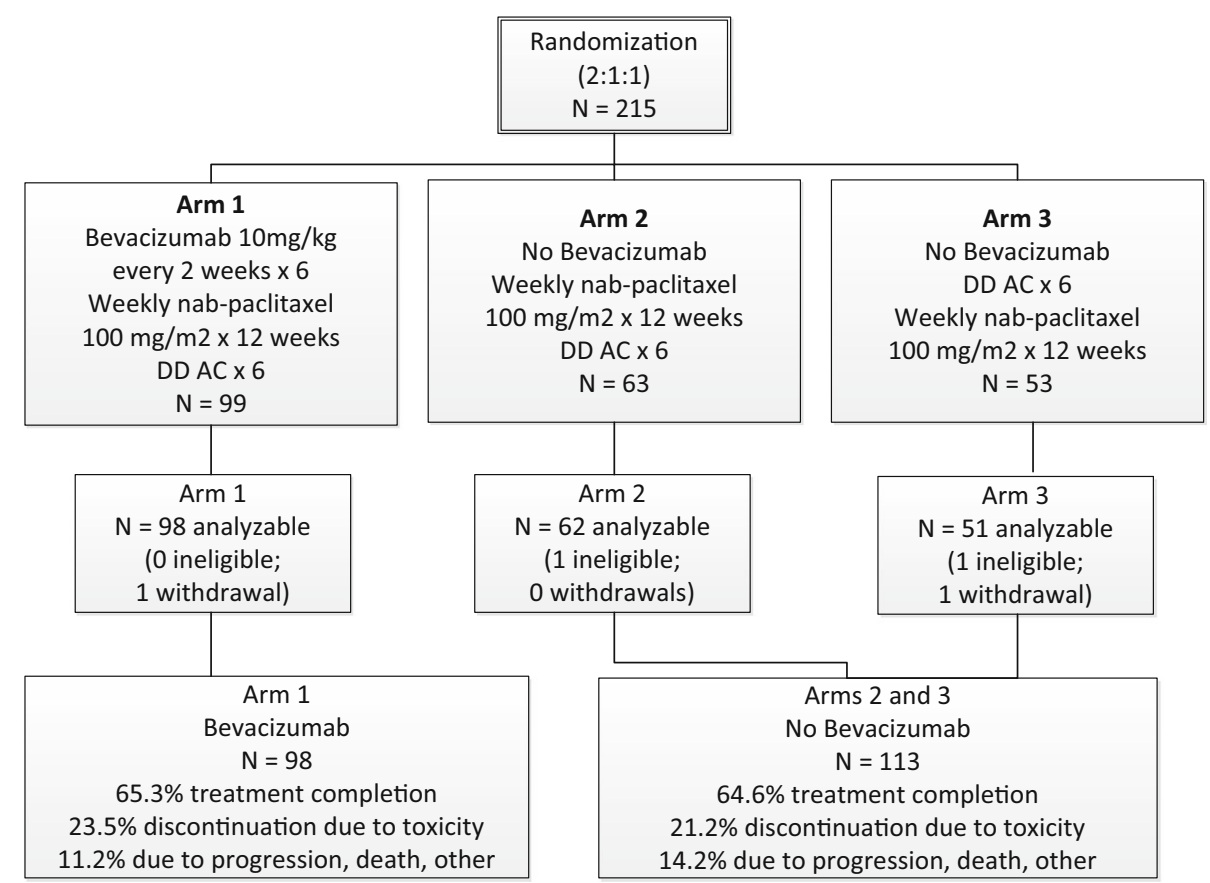

Table 1 Demographic and disease characteristics at randomization

\begin{tabular}{|c|c|c|c|}
\hline & $\begin{array}{l}\text { Arm 1 } \\
\text { Bevacizumab } \\
\text { Nab-paclitaxel } \\
\text { DD AC }\end{array}$ & $\begin{array}{l}\text { Arms } 2 \text { and } 3 \\
\text { No Bevacizumab } \\
\text { Nab-paclitaxel } \\
\text { DD AC }\end{array}$ & Total \\
\hline Randomized & 99 & 116 & 215 \\
\hline Ineligible or withdrew consent & $1(1.0 \%)$ & $3(2.6 \%)$ & $4(1.9 \%)$ \\
\hline Analyzed & 98 & 113 & 211 \\
\hline Age median (range) & $51.7(22-71)$ & $51.3(31-75)$ & $51.5(22-75)$ \\
\hline \multicolumn{4}{|l|}{ Race } \\
\hline White & $70(71.4 \%)$ & $84(74.3 \%)$ & $154(73.0 \%)$ \\
\hline Black & $20(20.4 \%)$ & $18(15.9 \%)$ & $38(18.0 \%)$ \\
\hline Asian/Pacific Islander & $5(5.1 \%)$ & $6(5.3 \%)$ & $11(5.2 \%)$ \\
\hline Other/unknown & $3(3.1 \%)$ & $5(4.4 \%)$ & $8(3.8 \%)$ \\
\hline \multicolumn{4}{|l|}{ IBC or Non-IBC LABC } \\
\hline IBC & $10(10.2 \%)$ & $14(12.4 \%)$ & $24(11.4 \%)$ \\
\hline Non-IBC LABC & $88(89.8 \%)$ & $99(87.6 \%)$ & $187(88.6 \%)$ \\
\hline \multicolumn{4}{|l|}{ Hormone receptor status } \\
\hline Positive: ER + or $\mathrm{PgR}+$ & $66(67.3 \%)$ & $78(69.0 \%)$ & $144(68.2 \%)$ \\
\hline Negative: $\mathrm{ER}-$ and $\mathrm{PR}-(\mathrm{TNBC})$ & $32(32.7 \%)$ & $35(31.0 \%)$ & $67(31.8 \%)$ \\
\hline \multicolumn{4}{|l|}{ Breast cancer stage (1 missing) } \\
\hline IIB & $35(35.7 \%)$ & $52(46.4 \%)$ & $87(41.4 \%)$ \\
\hline IIIA & $32(32.6 \%)$ & $30(26.8 \%)$ & $62(29.5 \%)$ \\
\hline IIIB & $29(29.6 \%)$ & $24(21.4 \%)$ & $53(25.2 \%)$ \\
\hline IIIC & $2(2.0 \%)$ & $6(5.4 \%)$ & $8(3.8 \%)$ \\
\hline
\end{tabular}

\section{Secondary end points}

Figure 3a shows the Kaplan-Meier comparison of OS. Thus far, there have been 31 deaths with a median follow- 
Table 2 Primary outcome of pathological complete response (pCR) by randomized arm

\begin{tabular}{|c|c|c|c|}
\hline & $\begin{array}{l}\text { Arm 1 } \\
\text { Bevacizumab } \\
\text { Nab-paclitaxel } \\
\text { DD AC } \\
N=98\end{array}$ & $\begin{array}{l}\text { Arms } 2 \text { and } 3 \\
\text { No Bevacizumab } \\
\text { Nab-paclitaxel } \\
\text { DD AC } \\
N=113\end{array}$ & Total \\
\hline \multicolumn{4}{|l|}{ Status at surgery postchemo } \\
\hline No surgery/incomplete report & $7(7.1 \%)$ & $10(8.9 \%)$ & $17(8.0 \%)$ \\
\hline Residual disease & $56(57.1 \%)$ & $79(69.9 \%)$ & $135(64.0 \%)$ \\
\hline Pathological complete response & $35(35.7 \%)$ & $24(21.2 \%)$ & $59(28.0 \%)$ \\
\hline \multicolumn{4}{|l|}{ Number with pCR (rates) } \\
\hline Overall & $35 / 98(35.7 \%)$ & 24/113 (21.2 \%) & $59 / 211(28.0 \%)$ \\
\hline IBC & $3 / 10(30.0 \%)$ & 2/14 (14.3\%) & $5 / 24(20.8 \%)$ \\
\hline Non-IBC LABC & $32 / 88(36.4 \%)$ & $22 / 99(22.2 \%)$ & $54 / 187(28.9 \%)$ \\
\hline ER/PgR-positive & $16 / 66(24.2 \%)$ & 14/78 (18.0\%) & $30 / 144(20.8 \%)$ \\
\hline TNBC & $19 / 32(59.4 \%)$ & $10 / 35(28.6 \%)$ & $29 / 67(43.3 \%)$ \\
\hline
\end{tabular}

up of about 3 years, with 3-year OS of 86 and $87 \%$ for the bevacizumab and non-bevacizumab groups, for a hazard ratio (HR) of 0.84 (95\% CI $0.41-1.73, p=0.64)$. When separated by hormone receptor status, there were no significant differences in OS between the bevacizumab and non-bevacizumab groups. The survival curves suggest a possible small benefit in the TNBC subset [Fig. 3b: $\mathrm{HR}=0.49$ (95 \% CI 0.19-1.29), log-rank $p=0.14]$ but not in the ER/PgR+ subset (Fig. 3c: HR $=1.85(95 \% \mathrm{CI}$ 0.58-5.85), log-rank $p=0.33$ ), but the interaction of hormone receptor status and bevacizumab for OS was not statistically significant $(p=0.14)$. In a landmarked analysis starting at 6 months (i.e., after surgery), having a pCR was highly associated with subsequent OS for hormone receptor-negative disease $\quad(\mathrm{HR}=0.15 ; \quad 95 \% \quad \mathrm{CI}$ 0.03-0.63), but not for hormone receptor-positive disease $(\mathrm{HR}=0.32 ; 95 \%$ CI 0.04-2.50).

Event-free survival (EFS) also showed no significant difference by treatment (Fig. 4a: $p=0.71 ; \mathrm{HR}=0.89$; $95 \%$ CI 0.48-1.65). When separated by hormone receptor status, there were no significant differences for EFS, but in TNBC there was a trend favoring the bevacizumab arm [Fig. 4b: $\mathrm{HR}=0.46 \quad(95 \%$ CI 0.20-1.05), log-rank $p=0.06]$ which was not seen in $\mathrm{ER} / \mathrm{PgR}+$ patients [Fig. 4c: $\mathrm{HR}=2.20 \quad(95 \% \quad \mathrm{CI}$ 0.84-5.78), log-rank $p=0.10]$, and the interaction of treatment and receptor status was statistically significant for $\operatorname{EFS}(p=0.028)$.

\section{Treatment delivery and toxicity}

Overall, Grade 3/4 events were common and did not differ between the bevacizumab and non-bevacizumab arms (bevacizumab $67 \%$; non-bevacizumab $65 \%$ ) (Table 3). Grade 4 toxicities were seen in $19(21 \%)$ of patients in the bevacizumab arm (two with sepsis, one with respiratory failure, one with bilateral pulmonary emboli and deep vein thrombosis, and 17 with hematologic events that included anemia, febrile neutropenia, or thrombocytopenia). On the other hand, 20 (19\%) patients in the non-bevacizumab arm experienced Grade 4 events (one with heart failure, infectious enterocolitis, sepsis, and respiratory failure, one with dyspnea, one with Grade 4 anemia and hypercalcemia, one with anemia and febrile neutropenia, and 17 with other hematologic toxicities).

\section{Discussion}

We demonstrate that the addition of bevacizumab to neoadjuvant chemotherapy significantly increased the pCR rate in patients with $\mathrm{LABC} / \mathrm{IBC}$ without significant additional toxicity, and that this increase was more pronounced in patients with TNBC. These data suggest that the addition of bevacizumab to anthracycline- and taxane-based chemotherapy enhances its cytotoxicity.

The addition of bevacizumab to chemotherapy in patients with metastatic breast cancer was initially approved by the FDA on the basis of improvements in response rate and $\mathrm{PFS}$, but this approval was withdrawn when these studies failed to demonstrate improvement in OS [40], while the European Medicines Agency (EMA) has approved its use with paclitaxel and capecitabine. Meanwhile, four large randomized trials, in addition to S0800, have investigated the addition of bevacizumab to neoadjuvant chemotherapy for breast cancer [40-43]. All have reported a significant benefit with the addition of bevacizumab to anthracycline/taxane-based chemotherapy (Table 4). As in our trial, GeparQuinto, CALGB 40603, and ARTemis demonstrated a significant increase in the pCR rate in patients with TNBC, while NSABP B-40 demonstrated a higher $\mathrm{pCR}$ rate with bevacizumab in ER/ 
A

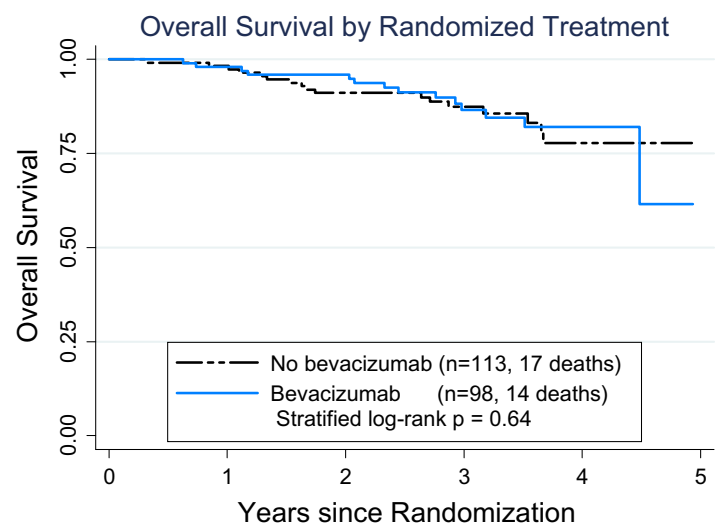

Number at risk

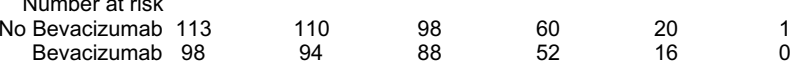

B
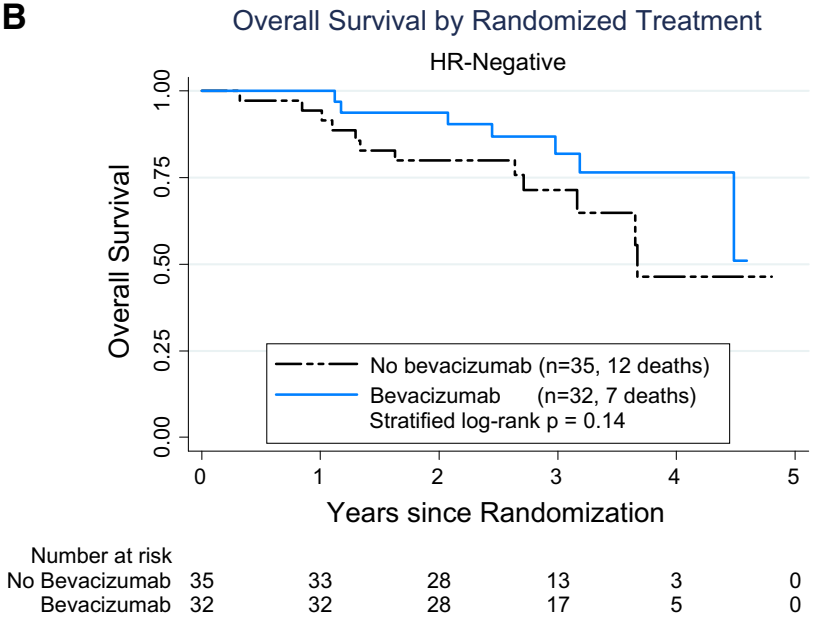

C

Overall Survival by Randomized Treatment

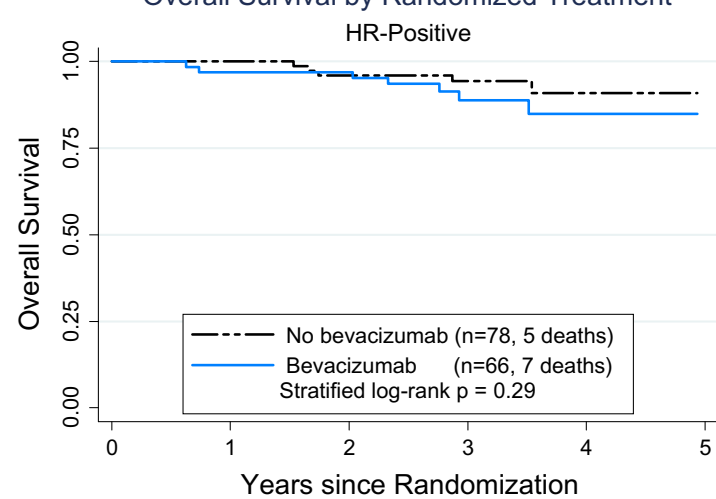

Number at risk No Bevacizumab 78 Bevacizumab 66

$\begin{array}{lllll}77 & 70 & 47 & 17 & 1 \\ 62 & 60 & 35 & 11 & 0\end{array}$

Fig. 3 Overall survival. Time from randomization to death due to any cause. a Overall survival for all patients. b Overall survival for patients with triple-negative (ER- and $\mathrm{PgR}-$ ) disease. c Overall survival for patients with $\mathrm{ER}+$ or $\mathrm{PgR}+$ disease

PgR + breast cancer but no statistically significant difference in TNBC.

Our EFS and OS results failed to demonstrate significant differences favoring the addition of bevacizumab for the
A

Event-Free Survival by Randomized Treatment

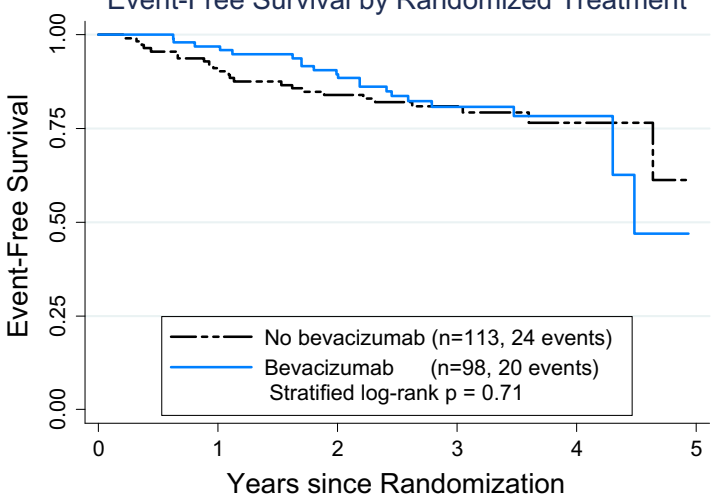

Number at risk

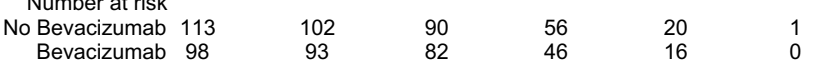

B

Event-Free Survival by Randomized Treatment

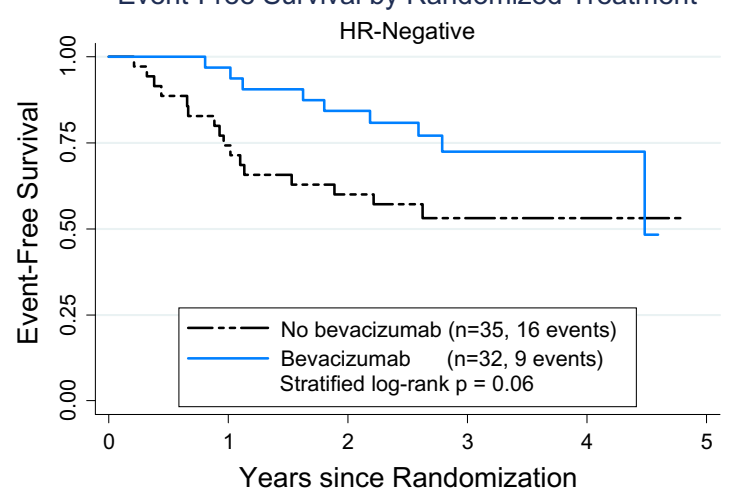

Number at risk

$\begin{array}{rllllll}\text { No Bevacizumab } & 35 & 26 & 21 & 10 & 3 & 0 \\ \text { Bevacizumab } & 32 & 31 & 25 & 14 & 5 & 0\end{array}$

C

Event-Free Survival by Randomized Treatment

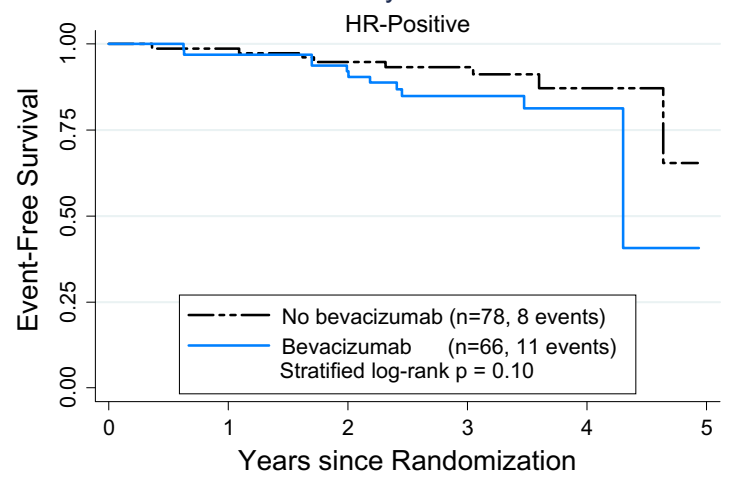

Number at risk

$\begin{array}{lllllll}\text { No Bevacizumab } & 78 & 76 & 69 & 46 & 17 & 1\end{array}$

$\begin{array}{lllllll}\text { Bevacizumab } & 66 & 62 & 57 & 32 & 11 & 0\end{array}$

Fig. 4 Event-free survival. Time from randomization to progression, recurrence, or death due to any cause. a Event-free survival for all patients. b Event-free survival for patients with triple-negative (ERand $\mathrm{PgR}-$ ) disease. c Event-free survival for patients with $\mathrm{ER}+$ or $\mathrm{PgR}+$ disease

overall study population, but in the relatively small $(n=67)$ TNBC subset, the EFS and OS hazard ratios trend in favor of the bevacizumab arm (HR 0.46, $p=0.06$ and HR $0.49, p=0.14)$, respectively, while the hazard ratios 
Table 3 Serious adverse events (Grades 3 and 4) by treatment arm

\begin{tabular}{|c|c|c|c|}
\hline & $\begin{array}{l}\text { Arm } 1 \\
\text { Bevacizumab } \\
\text { Nab-paclitaxel } \\
\text { DD AC } \\
N=95\end{array}$ & $\begin{array}{l}\text { Arms } 2 \text { and } 3 \\
\text { No Bevacizumab } \\
\text { Nab-paclitaxel } \\
\text { DD AC } \\
N=110\end{array}$ & Total \\
\hline Any event & $64(67 \%)$ & $69(63 \%)$ & $133(65 \%)$ \\
\hline ARDS & $1(1 \%)$ & $0(0 \%)$ & $1(1 \%)$ \\
\hline Diarrhea & $3(3 \%)$ & $2(2 \%)$ & $5(2 \%)$ \\
\hline Dyspnea & $3(3 \%)$ & $1(1 \%)$ & $4(2 \%)$ \\
\hline Enterocolitis, infectious & $0(0 \%)$ & $2(2 \%)$ & $2(1 \%)$ \\
\hline Hand foot syndrome & $1(1 \%)$ & $2(2 \%)$ & $3(1 \%)$ \\
\hline Heart failure & $0(0 \%)$ & $1(1 \%)$ & $1(1 \%)$ \\
\hline $\begin{array}{l}\text { Hematologic events (including anemia, } \\
\text { febrile neutropenia, thrombocytopenia) }\end{array}$ & $44(46 \%)$ & $39(35 \%)$ & $83(40 \%)$ \\
\hline Hypercalcemia & $0(0 \%)$ & $1(1 \%)$ & $1(1 \%)$ \\
\hline Hypertension & $7(7 \%)$ & $3(3 \%)$ & $10(5 \%)$ \\
\hline Nausea & $6(6 \%)$ & $9(8 \%)$ & $15(7 \%)$ \\
\hline Pain & $2(2 \%)$ & $4(4 \%)$ & $6(3 \%)$ \\
\hline Respiratory failure & $1(1 \%)$ & $1(1 \%)$ & $2(1 \%)$ \\
\hline Sepsis & $2(2 \%)$ & $1(1 \%)$ & $3(1 \%)$ \\
\hline Thromboembolic event & $1(1 \%)$ & $2(2 \%)$ & $3(1 \%)$ \\
\hline
\end{tabular}

for these endpoints trend negatively in the larger ER/ PGR+ population (HR 2.20, $p=0.10$ and HR 1.85, $p=0.33$, respectively). None of the neoadjuvant studies was powered to definitely demonstrate an EFS or OS benefit for the addition of bevacizumab, overall or in the TNBC population. The only study to show a survival benefit with bevacizumab was NSABP B-40, which was also the only one in which patients received bevacizumab in the adjuvant as well as the neoadjuvant settings; as with their pCR data, the survival benefit was seen only in the $\mathrm{ER} / \mathrm{PgR}+$ subset, and OS improvement was reported despite the absence of significant improvement in DFS [44]. The addition of bevacizumab also failed to improve DFS or OS in two sizable trials in the adjuvant setting $[19,20]$; however, these trials were comprised largely of lower risk patients, and in E5103 the improvement in DFS with bevacizumab for TNBC approached significance (HR $0.77,95 \%$ CI $0.58-1.03$ ).

These studies suggest that bevacizumab may be most helpful in patients with high-risk cancers, defined by both clinical stage and subtype. We have failed to identify any subset of breast cancer patients most likely to benefit from bevacizumab [22, 45]. In CALGB 40603, a randomized phase II limited to stage II-III TNBC, investigators studied how intrinsic subtype assigned by PAM50 and other gene signatures affected the impact of bevacizumab on pCR rates [46]. In basal-like cancers, the addition of bevacizumab significantly increased pCR in the breast (64 vs $45 \%$ ) and the breast/axilla (57 vs $43 \%$ ) rates, while paradoxically lowering pCR rates in relatively small $(12.7 \%)$ number of non-basal-like cancers, resulting in a significant interaction between subtype and bevacizumab-specific pCR benefit $(p=0.02)$. mRNA signatures for high proliferative rate, low estrogen signaling, and high TP53 mutation were also associated with greater pCR benefit with the addition of bevacizumab, suggesting that even within TNBC there are biologically defined patient subsets that may benefit differentially from this agent. Similar analyses are underway for S0800, and will be reported separately. If confirmed, these findings could suggest that identifying and excluding a biologically defined subset of 'bevacizumab-resistant' patients lead to positive DFS and OS results from studies of bevacizumab in the adjuvant or neoadjuvant settings.

We also hypothesize that bevacizumab may have had activity in our study because of the unique eligibility requirement for LABC/IBC. Several studies have shown that such extensive tumors with high levels of neoangiogenesis are more likely to benefit from the addition of bevacizumab to chemotherapy than early-stage cancers with lower levels of tumor vasculature [1, 8-11, 47]. Therefore, we propose that perhaps these patients are particularly susceptible to an antiangiogenic agent such as bevacizumab.

Our study has several limitations. The power of a trial to detect survival improvement from (neo) adjuvant chemotherapy is influenced by the subtype composition of 
Table 4 Neoadjuvant bevacizumab trials in HER2-negative breast cancer

\begin{tabular}{|c|c|c|c|c|c|}
\hline \multirow[t]{2}{*}{ Trial (definition of pCR) } & \multicolumn{3}{|l|}{$\mathrm{pCR}$} & \multirow[t]{2}{*}{ DFS } & \multirow[t]{2}{*}{ OS } \\
\hline & All $(\%)$ & ER/PgR positive $(\%)$ & ER and $\mathrm{PgR}$ negative $(\%)$ & & \\
\hline S0800 (ypT0, ypTis, ypN0) & & & & 3 years & 3 years \\
\hline Bevacizumab & 36 & 24 & 59 & $\begin{array}{l}\mathrm{HR}=0.89 \\
P=0.71\end{array}$ & $86 \%$ \\
\hline No bevacizumab & 21 & 18 & 29 & & $87 \%$ \\
\hline GeparQuinto [41] (ypT0, ypN0) & & & & 3 years & 3 years \\
\hline Bevacizumab & 18.4 & 7.7 & 39.9 & $80.8 \%$ & $90.7 \%$ \\
\hline No bevacizumab & 14.9 & 7.8 & 81.5 & $81.5 \%$ & $88.7 \%$ \\
\hline Artemis [40] (ypT0, ypTis, ypN0) & & & & Not reported & Not reported \\
\hline Bevacizumab & 22.0 & 6.0 & 45.0 & & \\
\hline No bevacizumab & 17.0 & 7.0 & 31.0 & & \\
\hline NSABP-B40 [44] (ypT0, any pN) & & & & 5 years & 5 years \\
\hline Bevacizumab & 34.5 & 23.2 & 51.1 & $\begin{array}{l}\mathrm{HR}=0.8 \\
P=0.06\end{array}$ & $\begin{array}{l}\mathrm{HR}=0.65 \\
P=0.004\end{array}$ \\
\hline No bevacizumab & 28.2 & 15.1 & 47.1 & & \\
\hline CALGB 40603 [43] (ypT0, any pN) & & & & Not reported & Not reported \\
\hline Bevacizumab & NA & NA & 59 & & \\
\hline No Bevacizumab & NA & NA & 48 & & \\
\hline
\end{tabular}

$E R$ estrogen receptor, $H R$ hazard ratio, $p C R$ pathologic complete response, $P G R$ progesterone receptor, $T N$ triple negative, $D F S$ disease-free survival, $O S$ overall survival, $N A$ not applicable

the accrued patients [48, 49]. Slightly more than twothirds of our patients had ER/PgR + cancers. Not only are these patients much less likely to achieve a pCR, they also have a better prognosis than TNBC and other more aggressive breast cancer subtypes. Nearly $80 \%$ of our $\mathrm{ER} / \mathrm{PgR}+$ patients, across all treatment arms, were alive and free of disease recurrence at 4 years; EFS was only $50 \%$ in TNBC patients assigned to the control arm, almost sufficient for demonstrating a significant benefit in a small cohort. Effective postneoadjuvant therapy, especially adjuvant endocrine therapy in $\mathrm{ER} / \mathrm{PgR}+$ cancers, improves outcomes in patients with residual disease and thus diminishes the impact of a more effective neoadjuvant regimen.

In summary, we find the trend favoring improvement in EFS in our high-risk TNBC subset encouraging and believing that the addition of bevacizumab to chemotherapy in the neoadjuvant setting for these patients warrants further investigation. While the role of bevacizumab remains uncertain due to the lack of OS improvement in several studies that included a high proportion of low- to moderate-risk patients, the consistent association between the addition of bevacizumab with higher response and pCR rates in metastatic and neoadjuvant trials is intriguing and underscores the importance of finding predictive biomarkers for this drug. Our results also suggest that it may worth re-evaluating the role of bevacizumab in locally advanced TNBC.
Funding The work was supported in part by the National Institutes of Health (NIH)/National Cancer Institute (NCI)/National Clinical Trials Network (NCTN) grants CA180888, CA180819, CA180821, CA180835, CA180830, CA180846, CA180801, CA180834; NIH/ NCI Community Oncology Research Program (NCORP) grants CA189954, CA189856, CA189971, CA189822, CA189952, CA189804, CA189817, CA189953, CA189858, CA189957, CA189872, CA189853; legacy NIH/NCI grants CA35119, CA52654, CA04919, CA37981, CA58416, CA16385; and in part by Genentech (Roche), Abraxis BioScience (Celgene), and Helomics ${ }^{\mathrm{TM}}$.

\section{Compliance with ethical standards}

Conflicts of interest All remaining authors have declared that they have no conflicts of interest to report.

Disclosure Employment: E. Perez: Genentech; Stock or other ownership: E. Perez: Genentech; D. Hayes: Oncimmune LLC, De Soto, KS, USA, and Inbiomotion, Barcelona, Spain; Consulting or advisory role: J. Gralow: Novartis, Roche/Genentech; G. Hortobagyi: Lilly USA, LLC, Celgene, Norvartis, Merck, Bayer and Peregrine Pharmaceuticals, Inc; H. Mirshahidi: Clovis Oncology, Boehringer; D. Hayes: Pfizer Global Advisory Board for pablociclib; W. Sikov: Abbvie, Celgene (uncompensated) Honoraria: L. Pusztai: Celgene; D. Hayes: Lecture/Honorarium as a Visiting Consultant for Lilly Oncology, Indianapolis, IN; W Sikov: Eisai, Celgen Research funding: J. Gralow: Novartis, Roche/Genentech; L. Pusztai/institution/clinical trial support: D. Hayes: Sponsored Clinical ResearchPrinciple or co-Investigator: Merrimack Pharmaceuticals, Inc. (Parexel Intl Corp), Eli Lilly Company, Janssen R\&D, LLC (Johnson \& Johnson), Veridex (Johnson \& Johnson), Puma Biotechnology, Inc., (subcontract Wash Univ St. Louis to Univ Mich), Pfizer, Astra Zeneca, Astra Zeneca; Travel, accommodations or expenses: L. Pusztai/self: Celgene Royalties: D. Hayes: Royalties from licensed technology: Janssen R\&D, LLC (Johnson \& Johnson); Patents: D. 
Hayes: Title: A method for predicting progression free and overall survival at each follow-up timepoint during therapy of metastatic breast cancer patients using circulating tumor cells. Filed 14 Mar 2005 with the European Patent Office, Netherlands. Application No./ Patent No. 05725638.0-1223-US2005008602. Applicant/Proprietor: Immunicon Corporation. Dr. Daniel F. Hayes is designated as inventor/coinventor. Title: Diagnosis and Treatment of Breast Cancer. Patent No.: US 8,790,878 B2. Date of Patent: Jul. 29, 2014. Applicant Proprietor: University of Michigan. Dr. Daniel F. Hayes is designated as inventor/coinventor. Title: Circulating Tumor Cell Capturing Techniques and Devices. Patent No.: US 8,951,484 B2. Date of Patent: Feb. 10, 2015. Applicant Proprietor: University of Michigan. Dr. Daniel F. Hayes is designated as inventor/coinventor.

Open Access This article is distributed under the terms of the Creative Commons Attribution-NonCommercial 4.0 International License (http://creativecommons.org/licenses/by-nc/4.0/), which permits any noncommercial use, distribution, and reproduction in any medium, provided you give appropriate credit to the original author(s) and the source, provide a link to the Creative Commons license, and indicate if changes were made.

\section{Appendix: Surgical procedure}

Identification of the tumor bed was done by placement of clips by core needle biopsy prior to treatment. However, in the absence of a clip, detailed information about the location (quadrant, clock face, distance from nipple) and the presence of any other identifiable features (e.g., calcifications) was required to identify the tumor bed. Axillary staging requirements were as noted previously. Also, a detailed procedure for evaluation of surgical specimens following neoadjuvant therapy was described. Specimens were oriented with sutures by the surgeon following removal. The surgeon and breast pathologist were required to confer to ensure optimal evaluation of the primary tumor site for possible pCR. (1) In cases showing significant clinical response, each specimen was inked using multiple colors to identify each face of the specimen and then sectioned into 3-5 $\mathrm{mm}$ slices. The sliced specimen was radiographed and a radiologist reviewed the films to determine the presence and extent of residual tumor. The pathologist examined the sliced specimen grossly to identify suspicious areas and noted their proximity to margins. The radiographic and pathological evaluation was discussed with the surgeon who decided whether additional margins should be obtained. Permanent paraffin sections of the suspicious areas and margins were obtained. The number of sections taken was based on the gross inspection, radiologic features, and size of the resection specimen. The entire radiographic abnormality as well as firm and suspicious-appearing breast tissue was submitted for histologic evaluation. In general, for non-palpable (clinical complete response) cases, at least 10-15 blocks were examined to assess the presence of residual microscopic disease; (2) in cases with residual palpable mass (partial clinical response or no response in the breast), the resection specimen was inked and sectioned into $3-5 \mathrm{~mm}$ slices. The pathologist examined the slices and determined the tumor size on gross evaluation and confirmed the tumor size by microscopic evaluation; (3) Evaluation of axillary lymph nodes regardless of response. All axillary lymph nodes were also carefully evaluated by serial gross sectioning. One or two representative histologic sections were evaluated for lymph nodes that contain grossly identifiable metastatic carcinoma. The lymph nodes that do not show grossly identifiable tumor were submitted for histologic evaluation in their entirety. One representative histologic section was evaluated per paraffin block.

\section{References}

1. Chia S, Swain SM, Byrd DR, Mankoff DA (2008) Locally advanced and inflammatory breast cancer. J Clin Oncol 26:786-790

2. Singletary SE, Allred C, Ashley P et al (2002) Revision of the American Joint Committee on Cancer staging system for breast cancer. J Clin Oncol 20:3628-3636

3. Edge SB, Compton CC (2010) The American Joint Committee on Cancer: the 7th edition of the AJCC cancer staging manual and the future of TNM. Ann Surg Oncol 17:1471-1474

4. Masuda H, Brewer TM, Liu DD et al (2014) Long-term treatment efficacy in primary inflammatory breast cancer by hormonal receptor- and HER2-defined subtypes. Ann Oncol 25:384-391

5. Guarneri V, Broglio K, Kau SW et al (2006) Prognostic value of pathologic complete response after primary chemotherapy in relation to hormone receptor status and other factors. J Clin Oncol 24:1037-1044

6. Fumagalli D, Bedard PL, Nahleh Z et al (2012) A common language in neoadjuvant breast cancer clinical trials: proposals for standard definitions and endpoints. Lancet Oncol 13:e240 e248

7. von Minckwitz G, Untch M, Blohmer JU et al (2012) Definition and impact of pathologic complete response on prognosis after neoadjuvant chemotherapy in various intrinsic breast cancer subtypes. J Clin Oncol 30:1796-1804

8. Kleer CG, van Golen KL, Merajver SD (2000) Molecular biology of breast cancer metastasis. Inflammatory breast cancer: clinical syndrome and molecular determinants. Breast Cancer Res 2:423-429

9. Shirakawa K, Kobayashi H, Sobajima J et al (2003) Inflammatory breast cancer: vasculogenic mimicry and its hemodynamics of an inflammatory breast cancer xenograft model. Breast Cancer Res 5:136-139

10. Sledge GW Jr, Rugo HS, Burstein HJ (2006) The role of angiogenesis inhibition in the treatment of breast cancer. Clin Adv Hematol Oncol 4:1-10 (quiz 11-12)

11. Wedam SB, Low JA, Yang SX et al (2006) Antiangiogenic and antitumor effects of bevacizumab in patients with inflammatory and locally advanced breast cancer. J Clin Oncol 24:769-777

12. Hurwitz H, Kabbinavar F (2005) Bevacizumab combined with standard fluoropyrimidine-based chemotherapy regimens to treat colorectal cancer. Oncology 69(Suppl 3):17-24

13. Presta LG, Chen H, O'Connor SJ et al (1997) Humanization of an anti-vascular endothelial growth factor monoclonal antibody for the therapy of solid tumors and other disorders. Cancer Res 57:4593-4599 
14. Miller KD (2003) E2100: a phase III trial of paclitaxel versus paclitaxel/bevacizumab for metastatic breast cancer. Clin Breast Cancer 3:421-422

15. Jain RK, Duda DG, Clark JW, Loeffler JS (2006) Lessons from phase III clinical trials on anti-VEGF therapy for cancer. Nat Clin Pract Oncol 3:24-40

16. Midgley R, Kerr D (2005) Bevacizumab-current status and future directions. Ann Oncol 16:999-1004

17. Jain RK (2002) Tumor angiogenesis and accessibility: role of vascular endothelial growth factor. Semin Oncol 29:3-9

18. Lenzer J (2011) FDA committee votes to withdraw bevacizumab for breast cancer. BMJ 343:d4244

19. Cameron D, Brown J, Dent R et al (2013) Adjuvant bevacizumab-containing therapy in triple-negative breast cancer (BEATRICE): primary results of a randomised, phase 3 trial. Lancet Oncol 14:933-942

20. Miller K, O'Neill AM, Dang CT et al (2014) Bevacizumab (Bv) in the adjuvant treatment of HER2-negative breast cancer: final results from Eastern Cooperative Oncology Group E5103. J Clin Oncol 32:5s (Suppl; abstr 500)

21. Hayes DF (2011) Bevacizumab treatment for solid tumors: boon or bust? JAMA 305:506-508

22. Jubb AM, Harris AL (2010) Biomarkers to predict the clinical efficacy of bevacizumab in cancer. Lancet Oncol 11:1172-1183

23. Sweeney CJ, Miller KD, Sissons SE et al (2001) The antiangiogenic property of docetaxel is synergistic with a recombinant humanized monoclonal antibody against vascular endothelial growth factor or 2-methoxyestradiol but antagonized by endothelial growth factors. Cancer Res 61:3369-3372

24. Desai N, Trieu V, Yao Z et al (2006) Increased antitumor activity, intratumor paclitaxel concentrations, and endothelial cell transport of cremophor-free, albumin-bound paclitaxel, ABI-007, compared with cremophor-based paclitaxel. Clin Cancer Res 12:1317-1324

25. Muller BG, Leuenberger H, Kissel T (1996) Albumin nanospheres as carriers for passive drug targeting: an optimized manufacturing technique. Pharm Res 13:32-37

26. Kim YW, Park YK, Lee J et al (1998) Expression of osteopontin and osteonectin in breast cancer. J Korean Med Sci 13:652-657

27. Van den Eynden GG, Van Laere SJ, Van der Auwera I et al (2006) Overexpression of caveolin-1 and -2 in cell lines and in human samples of inflammatory breast cancer. Breast Cancer Res Treat 95:219-228

28. Link JS, Waisman JR, Nguyen B, Jacobs CI (2007) Bevacizumab and albumin-bound paclitaxel treatment in metastatic breast cancer. Clin Breast Cancer 7:779-783

29. Volk LD, Flister MJ, Chihade D et al (2011) Synergy of nabpaclitaxel and bevacizumab in eradicating large orthotopic breast tumors and preexisting metastases. Neoplasia 13:327-338

30. Untch M, Jackisch C, Schneeweiss A et al (2015) A randomized phase III trial comparing neoadjuvant chemotherapy with weekly nanoparticle-based paclitaxel with solvent-based paclitaxel followed by anthracyline/cyclophosphamide for patients with early breast cancer (GeparSepto); GBG 69. Cancer Res 75(9 Suppl):S2-07

31. Wolff AC, Hammond ME, Schwartz JN et al (2007) American Society of Clinical Oncology/College of American Pathologists guideline recommendations for human epidermal growth factor receptor 2 testing in breast cancer. J Clin Oncol 25:118-145

32. Budd GT, Barlow WE, Moore HC et al (2015) SWOG S0221: a phase III trial comparing chemotherapy schedules in high-risk early-stage breast cancer. J Clin Oncol 33:58-64

33. Hutchins LF, Green SJ, Ravdin PM et al (2005) Randomized, controlled trial of cyclophosphamide, methotrexate, and fluorouracil versus cyclophosphamide, doxorubicin, and fluorouracil with and without tamoxifen for high-risk, node-negative breast cancer: treatment results of Intergroup Protocol INT-0102. J Clin Oncol 23:8313-8321

34. Martin M, Segui MA, Anton A et al (2010) Adjuvant docetaxel for high-risk, node-negative breast cancer. $N$ Engl J Med 363:2200-2210

35. Blum JL, Savin MA, Edelman G et al (2007) Phase II study of weekly albumin-bound paclitaxel for patients with metastatic breast cancer heavily pretreated with taxanes. Clin Breast Cancer 7:850-856

36. Recht A, Edge SB, Solin LJ et al (2001) Postmastectomy radiotherapy: clinical practice guidelines of the American Society of Clinical Oncology. J Clin Oncol 19:1539-1569

37. Ellis GK, Barlow WE, Gralow JR et al (2011) Phase III comparison of standard doxorubicin and cyclophosphamide versus weekly doxorubicin and daily oral cyclophosphamide plus granulocyte colony-stimulating factor as neoadjuvant therapy for inflammatory and locally advanced breast cancer: SWOG 0012 . J Clin Oncol 29:1014-1021

38. Green S, Benedetti J, Smith A (2012) Clinical trials in oncology, 3rd edn. CRC Press, Bocca Raton

39. Yardley DA (2013) nab-Paclitaxel mechanisms of action and delivery. J Control Release 170:365-372

40. Earl HM, Hiller L, Dunn JA et al (2015) Efficacy of neoadjuvant bevacizumab added to docetaxel followed by fluorouracil, epirubicin, and cyclophosphamide, for women with HER2-negative early breast cancer (ARTemis): an open-label, randomised, phase 3 trial. Lancet Oncol 16:656-666

41. von Minckwitz G, Eidtmann H, Rezai M et al (2012) Neoadjuvant chemotherapy and bevacizumab for HER2-negative breast cancer. N Engl J Med 366:299-309

42. Bear HD, Tang G, Rastogi P et al (2012) Bevacizumab added to neoadjuvant chemotherapy for breast cancer. N Engl J Med 366:310-320

43. Sikov WM, Berry DA, Perou CM et al (2015) Impact of the addition of carboplatin and/or bevacizumab to neoadjuvant onceper-week paclitaxel followed by dose-dense doxorubicin and cyclophosphamide on pathologic complete response rates in stage II to III triple-negative breast cancer: CALGB 40603 (Alliance). J Clin Oncol 33:13-21

44. Bear HD, Tang G, Rastogi P et al (2015) Neoadjuvant plus adjuvant bevacizumab in early breast cancer (NSABP B-40 [NRG Oncology]): secondary outcomes of a phase 3, randomised controlled trial. Lancet Oncol 16:1037-1048

45. Lambrechts D, Lenz HJ, de Haas S et al (2013) Markers of response for the antiangiogenic agent bevacizumab. J Clin Oncol 31:1219-1230

46. Sikov WM, Barry WT, Hoadley KA et al (2015) Impact of intrinsic subtype by PAM50 and other gene signatures on pathologic complete response (pCR) rates in triple-negative breast cancer (TNBC) after neoadjuvant chemotherapy (NACT) plus/- carboplatin (Cb) or bevacizumab (Bev): CALGB 40603/150709 (Allianc). Cancer Res 75(9 Suppl):S4-05

47. Tredan O, Lacroix-Triki M, Guiu S et al (2015) Angiogenesis and tumor microenvironment: bevacizumab in the breast cancer model. Target Oncol 10:189-198

48. Kaufmann M, von Minckwitz G, Mamounas EP et al (2012) Recommendations from an international consensus conference on the current status and future of neoadjuvant systemic therapy in primary breast cancer. Ann Surg Oncol 19:1508-1516

49. Korn EL, Sachs MC, McShane LM (2015) Statistical controversies in clinical research: assessing pathologic complete response as a trial-level surrogate end point for early-stage breast cancer. Ann Oncol 27(1):10-15 